\title{
Fairness Enhancement Scheme for Multimedia Applications in IEEE 802.11e Wireless LANs
}

\author{
Young-Woo Nam, Sunmyeng Kim, and Si-Gwan Kim \\ Department of Computer Software Engineering \\ Kumoh National Institute of Technology \\ Gumi, South Korea
}

\begin{abstract}
Multimedia traffic should be transmitted to a receiver within the delay bound. The traffic is discarded when breaking its delay bound. Then, QoS (Quality of Service) of the traffic and network performance are lowered. The IEEE 802.11e standard defines a TXOP (Transmission Opportunity) parameter. The TXOP is the time interval in which a station can continuously transmit multiple data packets. All stations use the same TXOP value in the IEEE 802.11e standard. Therefore, when stations transmit traffic generated in different multimedia applications, fairness problem occurs. In order to alleviate the fairness problem, we propose a dynamic TXOP control scheme based on the channel utilization of network and multimedia traffic quantity in the queue of a station. The simulation results show that the proposed scheme improves fairness and QoS of multimedia traffic.
\end{abstract}

Keywords-fairness; multimedia traffic; EDCA; QoS; TXOP.

\section{INTRODUCTION}

The IEEE 802.11 wireless LAN is widely used for wireless access due to its easy deployment and low cost. The IEEE 802.11 standard defines a medium access control (MAC) protocol for sharing the channel among stations [1]. The distributed coordination function (DCF) was designed for a contention-based channel access.

The widespread use of multimedia applications requires new features such as high bandwidth and small average delay in wireless LANs. Unfortunately, the IEEE 802.11 MAC protocol cannot support quality of service (QoS) requirements $[2,3]$. In order to support multimedia applications with tight QoS requirements in the IEEE 802.11 MAC protocol, the IEEE 802.11e has been standardized [4]. It introduces a contentionbased new channel access mechanism called enhanced distributed channel access (EDCA). The EDCA supports the QoS by introducing four access categories (ACs). To differentiate the ACs, the EDCA uses a set of AC specific parameters, which include minimum contention window $C W \min [i]$, maximum contention window $C W \max [i]$, and arbitration interframe space (AIFS) AIFS $i]$ for $\mathrm{AC} i(i=0, \ldots$ , 3). Furthermore, the EDCA introduces a transmission opportunity (TXOP). The TXOP is the time interval in which a station has the right to initiate transmission. In other words, a station can transmit multiple data packets consecutively until the duration of transmission exceeds the specific TXOP limit. The TXOP provides not only service differentiation among various ACs, but also improves the network performance.
In the original TXOP of the EDCA, the TXOP limits at stations are fixed and generally allocated among stations with identical traffic load. Under this condition, fair bandwidth allocation is expected. However, if stations transmit data packets with different traffic load, fairness problem arises. This problem is explained in detail in Section II.

In order to support multimedia traffic, many schemes have been proposed in the literature. However, the previous schemes still have several problems. First, some of them need modifications to the IEEE 802.11e standard [5-8]. Therefore, they are not backward compatible with the legacy EDCA. For example, Deng et al. proposed a surplus TXOP diverter (STXD) scheme to define the TXOP limit for per-flow but not for per-ACs [6]. However, the standard is on a per-AC basis. Second, some use analytical models to calculate the QoS metrics which are usually derived based on a few impractical hypotheses. They do not reflect the characteristics of multimedia traffic. Therefore, they are always inaccurate and clearly not applicable to realistic environments $[9,10]$. Third, some require feedback information from stations to consider the dynamic behavior of multimedia flows, but the feedback cannot provide an appropriate indication to the current network load conditions in a real-time manner [11, 12]. Finally, others proposed very simple schemes to allocate the TXOP limit. A threshold-based dynamic (TBD) TXOP scheme dynamically adjusts the TXOP limit according to the queue length and the pre-setting threshold [13]. Each station has two TXOP limit values: a low and a high TXOP. If the queue length is below the threshold, the TXOP limit is fixed at the low value; otherwise, the TXOP limit is set to the high value. A distributed optimal (DO) TXOP scheme proposed in [14] uses the throughput information instead of the queue length. In the DO TXOP scheme, each station measures its throughput and compares it with the target throughput. If the measured throughput is higher than the target value, the station reduces its TXOP limit; otherwise, it increases its TXOP. It is hard for the stations in the TBD and DO TXOP schemes to have adequate TXOP limit since the both schemes allocate the TXOP limit based on only one parameter: the pre-setting threshold in the TBD TXOP scheme and the target throughput in the DO TXOP scheme. And the TBD and DO TXOP schemes do not take into account the channel utilization. Therefore, at high loads, all the stations in the both schemes have large TXOP limit. On the contrary, at light loads, all the stations have small TXOP limit. 
In this paper, we propose a simple and effective scheme for alleviating the fairness problem. The proposed scheme dynamically adjusts the TXOP limit based on the local information such as the channel utilization at QoS access point (QAP) and current network load at stations without any feedback information. Therefore, we call the proposed scheme DTC (Dynamic TXOP Control) scheme.

The paper is organized as follows. The fairness problem is presented in Section II. In Section III, the proposed DTC scheme is explained in detail. In Section IV, we discuss simulation results. Finally, we conclude in Section V.

\section{FAIRNESS PROBLEM}

All stations use the same TXOP limit in the IEEE 802.11e EDCA. If traffic quantity of each station is same, no problem occurs, because bandwidth is allocated fairly. If each station supports multimedia application service with different traffic generation rate, fairness problem occurs. As traffic generation rate is different, each station has mutually different traffic quantity. If all stations use the same TXOP limit value in this situation, a station with less multimedia traffic quantity can promptly transmit data packets in its queue. Thus, the station acquires good performance by satisfying its delay bound. However, a station with more multimedia traffic quantity performs backoff process in several times, which lengthens waiting time to transmit packets. As the delay bound of packets is not satisfied, a receiver discards them, thereby lowering performance of multimedia traffic. Thus, stations with less traffic quantity always have better performance than those with more traffic quantity. This causes fairness problem among stations with different traffic quantity.

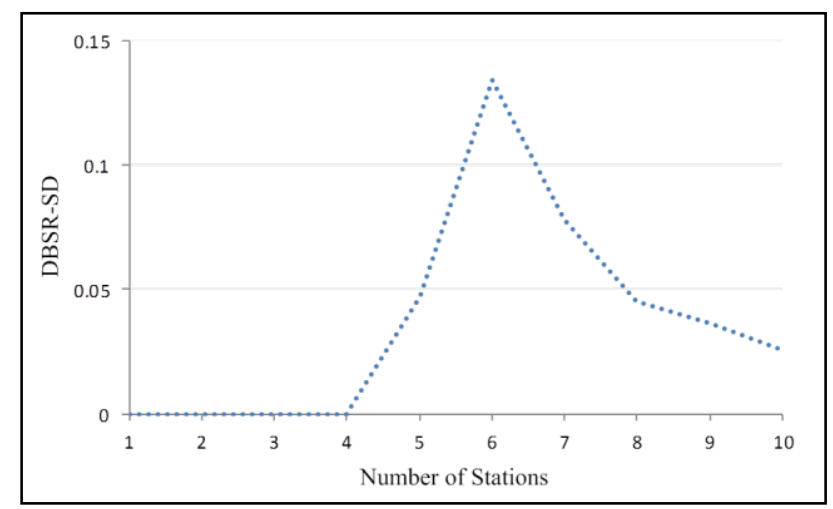

Figure 1. Standard deviation of delay bound success ratio according to the number of stations

Fig. 1 shows the standard deviation of delay bound success ratio for the IEEE 802.11e EDCA where all stations have the same TXOP limit. We simulated by using the simulation parameters in Tables I and II of Section IV. Delay bound success ratio is the number of data packets successfully transmitted to a receiver over the total number of transmitted data packets. The standard deviation hardly varies when there are few stations in the figure, since small traffic quantity can be immediately transmitted, regardless of traffic quantity in each station. When there are more than 4 stations, however, the standard deviation rapidly increases. This is because the delay bound success ratio becomes lower for elongated waiting time of packets in the queue of stations with more multimedia traffic quantity. When there are more than 6 stations, the standard deviation decreases, because the delay bound success ratio of all stations is lowered due to channel congestion. Like this, the provided QoS varies, depending on the multimedia traffic quantity of each station.

\section{DTC (DYNAMIC TXOP CONTROL) SCHEME}

The proposed DTC scheme dynamically adjusts TXOP limit value in consideration of channel utilization and queue utilization to alleviate the fairness problem depending on the traffic quantity. When the channel utilization of network is low, the DTC scheme can transmit more data packets. Then channel contention gets lower, if higher TXOP limit is allocated to a station with many packets in the queue. Thus, the possibility of packet collision becomes lower and channel waste can be reduced to improve overall network performance. The QoS of multimedia traffic may be lowered because a station with less data packets in the queue uses relatively lower TXOP limit. However, the DTC scheme is still effective since fair QoS can be provided irrespective of difference in traffic quantity.

The proposed DTC scheme is made up of two processes. First, QAP calculates the TXOP limit based on the channel utilization of network and then transmits it to stations through a beacon frame. Second, a station calculates the TXOP limit to be actually used to transmit data packets based on its queue utilization and the TXOP limit obtained from the beacon frame. Hereinafter the former is referred to as $T X O P_{Q A P}$ and the latter, as TXOP $P_{S T A}$ to distinguish between TXOP limit calculated by QAP and TXOP limit calculated by a station.

\section{A. Process to Calculate TXOP limit at QAP}

The channel utilization of network is used to calculate TXOP limit at QAP. Channel utilization is calculated by dividing the busy time of channel by a beacon frame transmission period. Busy time means time when channel is used, whether packets are successfully transmitted or not.

QAP measures channel busy time (Busy) using the carrier sensing during a beacon frame transmission period. Channel utilization $\left(C_{-} U t i l\right)$ is calculated as follows.

$$
\text { C_Util }=\frac{\text { Busy }}{\text { BeaconPeriod }}
$$

where BeaconPeriod indicates the period of a beacon frame.

As the channel utilization calculated in (1) fluctuates very irregularly in each calculation, performance fluctuates considerably if the calculated value is used as it is. Since it cannot be used in TXOP limit calculation as it is, moving average window is used as follows.

$$
C_{-} U t i l_{n}=(1-\alpha) \cdot C_{-} U t i l_{n-1}+\alpha \cdot C_{-} U t i l_{\text {current }}
$$

where $C_{-} U t i l_{\text {current }}$ is the channel utilization measured in the $n$th beacon frame period, $C_{-} U t i l_{n-1}$ and $C_{-} U t i l_{n}$ are the average channel utilizations at the end of $n-1$ th and $n$th beacon frame period, respectively. $\alpha$ is a smoothing factor. 
We introduce 4 new parameters to calculate $T X O P_{Q A P}$ : TXOP limit maximum value (TXOP $\left.{ }_{Q A P \max }\right)$ and minimum value $\left(T X O P_{Q A P \min }\right)$ at QAP, and upper threshold of channel utilization $\left(C_{-} U t i l_{\text {high }}\right)$ and lower threshold $\left(C_{-} U t i l_{\text {low }}\right)$.

Channel utilization increases and converges to the maximum utilization as the number of stations increases as shown in Fig. 2. As the number of stations increases, so does the possibility of packet collision. Thus, channel utilization increases due to frequent retransmission of packets.

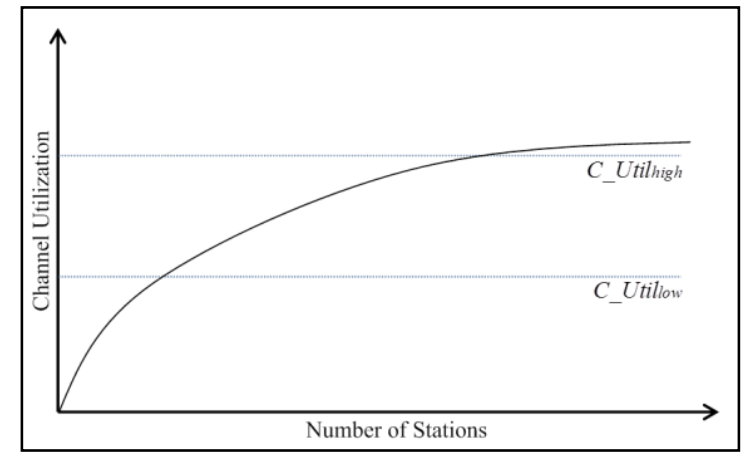

Figure 2. Channel utilization according to the number of stations

High channel utilization means that data packets are continuously transmitted as many stations contend for channel. Therefore, network performance needs to be improved by reducing TXOP limit. If channel utilization is low, TXOP limit should be increased so that a station can transmit many packets in a backoff process. Thus, $T X O P_{Q A P}$ is set to TXOP ${ }_{Q A P \min }$, if the measured channel utilization is larger than the upper threshold. If it is smaller than the lower threshold on the contrary, TXOP $P_{Q A P}$ is set to TXOP $P_{Q A P \max }$. When it is between the upper and lower thresholds, QAP calculates $T X O P_{Q A P}$ value as follows by using the channel utilization obtained from (1) and (2).

$$
\begin{aligned}
T X O P_{\text {diff }}= & T X O P_{Q A P \max }-T X O P_{Q A P m i n} . \\
T X O P_{Q A P}= & T X O P_{\text {diff }} \cdot \frac{C_{-} U t i l_{\text {high }}-C_{-} U t i l}{C_{-} U t i l_{\text {high }}-C_{-} U t i l_{\text {low }}} \\
& +T X O P_{Q A P \min } .
\end{aligned}
$$

$T X O P_{Q A P \min }$ is added to (4) so as to ensure that the calculated $T X O P_{Q A P}$ is always larger than $T X O P_{Q A P \min }$.

In Fig. 2, the upper threshold of channel utilization similar to maximum value is selected to fully use channel. The lower threshold is selected at medium value instead of low value, because no fairness problem occurs when channel utilization is low since traffic of all stations can be transmitted within their delay bound. We will explain how to decide these values in Section IV.

QAP transmits $T X O P_{Q A P}$ value obtained in the above process to all stations through a beacon frame. The process to calculate $T X O P_{Q A P}$ value based on the channel utilization at QAP is described in Fig. 3.

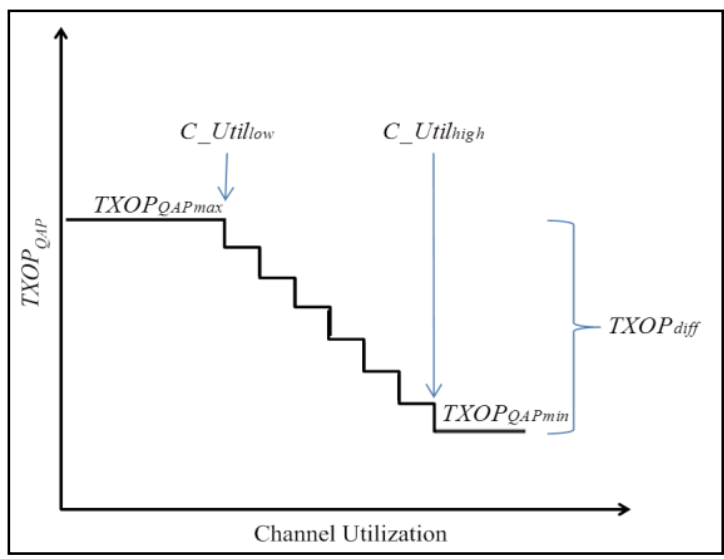

Figure 3. Calculation process of TXOP limit at QAP

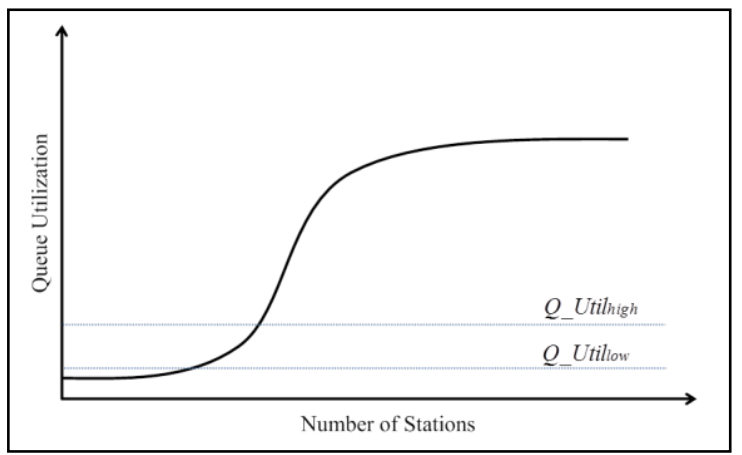

Figure 4. Queue utilization according to the number of stations

\section{B. Process to Calculate TXOP limit at a Station}

This subsection explains the process to calculate $T X O P_{S T A}$ to be used by each station. $T X O P_{S T A}$ is calculated based on the queue utilization of a station and $T X O P_{Q A P}$ obtained from a beacon frame transmitted by QAP.

Each station calculates queue utilization $\left(Q_{-}\right.$Util) after receiving a beacon frame. The utilization shows the quantity of data packets in the queue of a station. It is calculated as follows.

$$
Q_{-} U t i l=\frac{Q_{\text {packet }}}{Q_{\text {size }}}
$$

where $Q_{\text {size }}$ is the maximum number of packets that can be kept in the queue and $Q_{\text {packet }}$ is the number of packets in the queue.

Similar to the channel utilization calculation, queue utilization is calculated as follows by using moving average window to reflect traffic pattern.

$$
Q_{-} U t i l_{n}=(1-\alpha) \cdot Q_{-} U t i l_{n-1}+\alpha \cdot Q_{-} U t i l_{\text {current }}
$$

where $Q_{-} U t i l_{\text {current }}$ is the queue utilization measured after receiving $n$th beacon frame, $Q_{-} U t i l_{n-1}$ and $Q_{-} U t i l_{n}$ are the average queue utilizations after receiving $n-1$ th and $n$th beacon frame, respectively.

Fig. 4 shows the queue utilization depending on the increase of station number. In the figure, as the number of stations increases, queue utilization rapidly increases and 
converges to maximum size of queue. When there are many stations, the collision probability and the time to wait for transmission become bigger. Then, newly generated traffic exceeds the quantity of packets completely transmitted. Thus, the quantity of packets waiting for transmission in queue increases. Further, packets generated in excess of maximum size of the queue are discarded.

We introduce 3 new parameters to calculate TXOP $P_{S T A}$ : upper threshold $\left(Q_{-} U t i l_{h i g h}\right)$ of queue utilization and lower threshold ( $Q_{-} U t i l_{\text {low }}$ ), and TXOP limit minimum value $\left(T X O P_{S T A m i n}\right)$ at a station.

Each station calculates $T X O P_{S T A}$ value by using queue utilization acquired from (5) and (6). This process is similar to the process where QAP calculates $T X O P_{Q A P}$ value by using channel utilization. Since high queue utilization means that queue currently has many data packets, the delay bound of packets should be satisfied by transmitting the packets fast by increasing TXOP limit. Thus, $T X O P_{S T A}$ is set to TXOP ${ }_{Q A P}$ if the measured queue utilization is larger than the upper threshold. If it is smaller than the lower threshold on the contrary, TXOP $P_{S T A}$ is set to TXOP $P_{\text {STAmin }}$. If it is between the upper and lower thresholds, it is calculated as follows.

$$
\begin{aligned}
T X O P_{\text {diff }}= & T X O P_{Q A P}-T X O P_{S T A m i n} . \\
T X O P_{S T A}= & T X O P_{\text {diff }} \cdot \frac{Q_{-} U t i l_{\text {high }}-Q_{-} U t i l}{Q_{-} U t i l_{\text {high }}-Q_{-} U t i l_{\text {low }}} \\
& +T X O P_{S T A m i n} .
\end{aligned}
$$

In (8), $T X O P_{\text {STAmin }}$ is added to ensure that the calculated $T X O P_{S T A}$ is always larger than $T X O P_{\text {STAmin }}$.

In Fig. 4, there is not large difference between the upper and lower threshold values of queue utilization. Unless a station transmits all the packets in its queue within a given TXOP limit, queue utilization increases continuously. Thus, the upper threshold value should be set low to enable a station to have large TXOP limit. Then, the station can transmit packets fast. We will explain how to decide these values in Section IV.

The process where a station calculates $T X O P_{S T A}$ depending on queue utilization is described in Fig. 5.

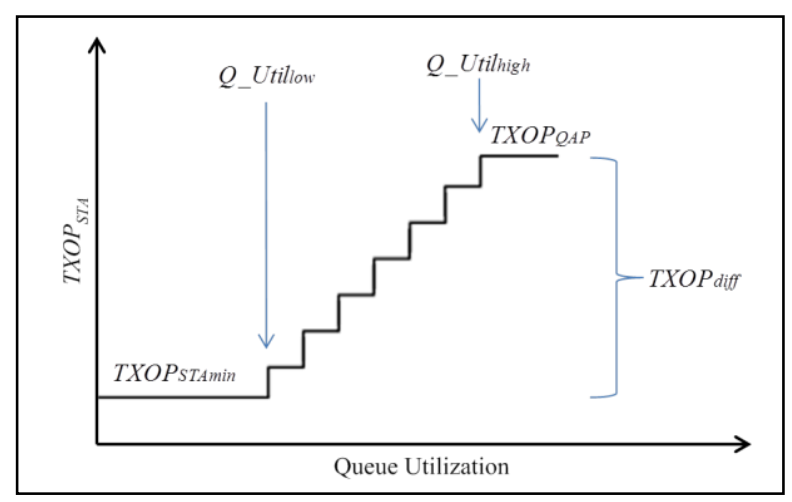

Figure 5. Calculation process of TXOP limit at a station

\section{Performance Evaluation}

Let us discuss the simulation results of the proposed DTC scheme. To validate the proposed scheme, we compare them to the results of the IEEE 802.11e standard EDCA. The parameters used in the simulation are listed in Table I. The average is calculated after repeating simulation for 30 seconds 10 times. Although TXOP limit value is given as time interval in the IEEE 802.11e standard, here it is represented in terms of the number of data packets. Thus, TXOP $P_{\text {STAmin }}$ value for the proposed DTC scheme is set to 2 data packets, TXOP $P_{Q A P \max }$ value is set to 10 data packets and TXOP $Q A P \min$ value is set to 8 data packets. On the contrary, fixed 5-data packet time is set for the IEEE 802.11e EDCA. 0.9 is used as smoothing factor. The delay bound of multimedia data packets is set to $33 \mathrm{~ms}$. Unless a packet is transmitted to a receiver within $33 \mathrm{~ms}$, it is discarded.

TABLE I. SIMULATION PARAMETERS

\begin{tabular}{|l|c|}
\hline \multicolumn{1}{|c|}{ Parameter } & Value \\
\hline Simulation Time & $30 \mathrm{~s}$ \\
\hline Beacon Period & $100 \mathrm{~ms}$ \\
\hline TXOP $P_{\text {QAPmin }}$ & 8 \\
\hline TXOP ${ }_{\text {QAPmax }}$ & 10 \\
\hline TXOP $P_{\text {STAmin }}$ & 2 \\
\hline Delay Bound & $33 \mathrm{~ms}$ \\
\hline$Q_{-}$Util $l_{\text {high }}$ & 0.20 \\
\hline$Q_{-}$Util $l_{\text {low }}$ & 0.05 \\
\hline Queue Size & 100 \\
\hline Smoothing Factor & 0.9 \\
\hline$C_{-}$Util $l_{\text {high }}$ & 0.95 \\
\hline$C_{-}$Util low & 0.75 \\
\hline
\end{tabular}

A constant data packet size of 1500 bytes is used. We use the negative exponential distribution to get the lengths of the data packet inter-arrival times. The average inter-arrival time of the distribution with arrival rate parameter $\lambda$ is $1 / \lambda$.

TABLE II. MULTIMEDIA DATA RATE PER STATION

\begin{tabular}{|l|c|c|}
\hline & Inter-arrival Time $(\boldsymbol{\mu s})$ & Data Rate(Mbps) \\
\hline Group 1 & 2326 & 5.16 \\
\hline Group 2 & 1587 & 7.56 \\
\hline
\end{tabular}

The number of stations used in the simulation is $1 \sim 10$ and the stations are divided into 2 groups by half. $\lambda$ is set as shown in Table II to differently set multimedia traffic quantity to be transmitted by stations belonging to each group. The average inter-arrival time of stations in group 1 is set to $2326 \mu \mathrm{s}(\lambda=$ $0.00043)$. Thus, these stations generate data packets at a rate of $5.16 \mathrm{Mbps}$. The average inter-arrival time of stations in group 2 is $1587 \mu \mathrm{s}(\lambda=0.00063)$ and data generation rate is $7.56 \mathrm{Mbps}$.

The following performance metrics are used to compare and analyze the results of simulation. 
- Channel Utilization: the fraction of time that the channel is used to transmit data packets.

- Normalized Throughput: the amount of useful data successfully transmitted divided by the capacity of the medium.

- DBSR (Delay Bound Success Ratio): the ratio of the number of packets which are successfully transmitted without breaking their delay bound to the number of all data packets transmitted to a receiver.

- DBSR-SD (Standard Deviation of Delay Bound Success Ratio): the standard deviation of DBSR.

- Queue Utilization: the ratio of the number of packets in the queue to the queue size.

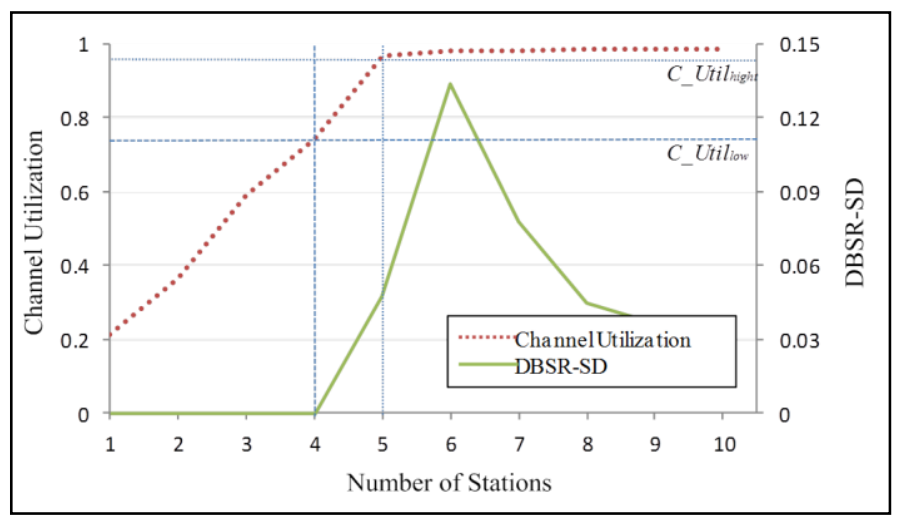

Figure 6. Channel utilization and DBSR-SD according to the number of stations

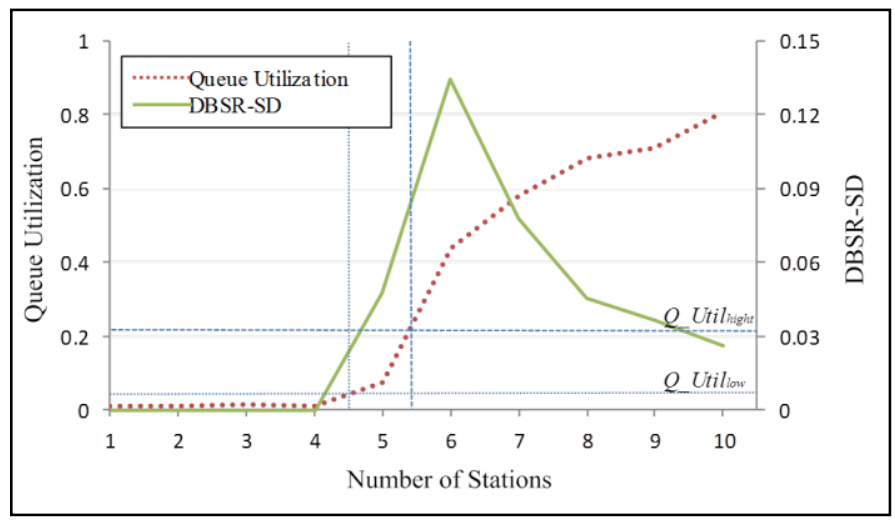

Figure 7. Queue utilization and DBSR-SD according to the number of stations

The values of $C_{-} U t i l_{\text {low }}$ and $C_{-} U t i l_{\text {high }}$ are decided by using the simulation results for TXOP limit of the IEEE 802.11e EDCA. Fig. 6 shows the results of channel utilization and DBSR-SD according to the number of stations. $C_{-} U t i l_{\text {low }}$ value is set to 0.75 , the point where DBSR-SD increases and the fairness problem occurs when there are more than 4 stations. $C_{-} U t i l_{\text {high }}$ value is set to 0.95 , the point where channel utilization begins to converge to maximum value.

The values of $Q_{-} U t i l_{\text {low }}$ and $Q_{-} U t i l_{\text {high }}$ are decided by using the results acquired in the same environment as Fig. 6.
Fig. 7 shows the queue utilization and the DBSR-SD caused by increasing the number of stations. $Q_{-} U t i l_{\text {low }}$ value is set to 0.05 , the point where a station cannot transmit all the packets in its queue during one TXOP limit, and $Q_{-} U t i l_{h i g h}$ is set to 0.2 , because DBSR-SD rapidly increases in the interval where the number of stations change from 5 to 6 .

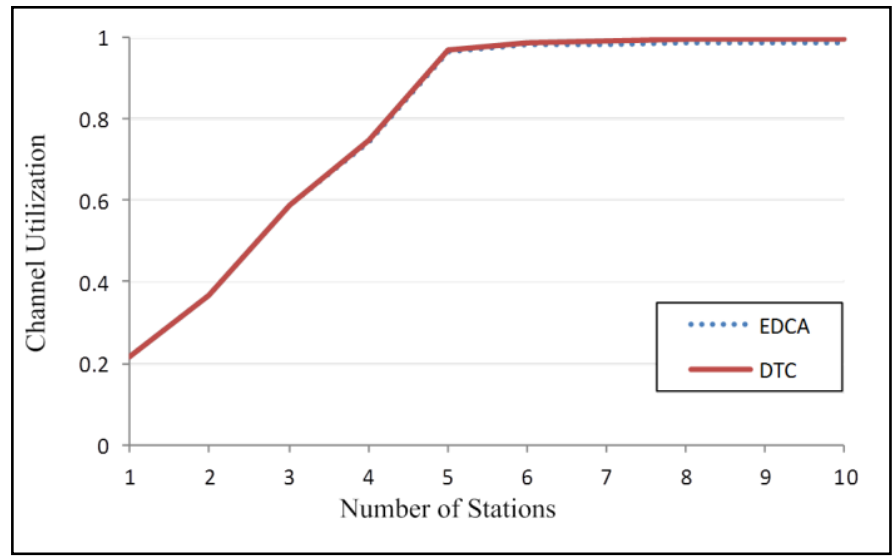

Figure 8. Channel utilization according to the number of stations

Fig. 8 shows channel utilization of the IEEE 802.11e EDCA and the proposed DTC scheme according to the number of stations. The figure shows that both of EDCA and DTC have similar channel utilization irrespective of the number of stations. The transmitted traffic does not fully use the capacity of media until the number of stations reaches 5 .

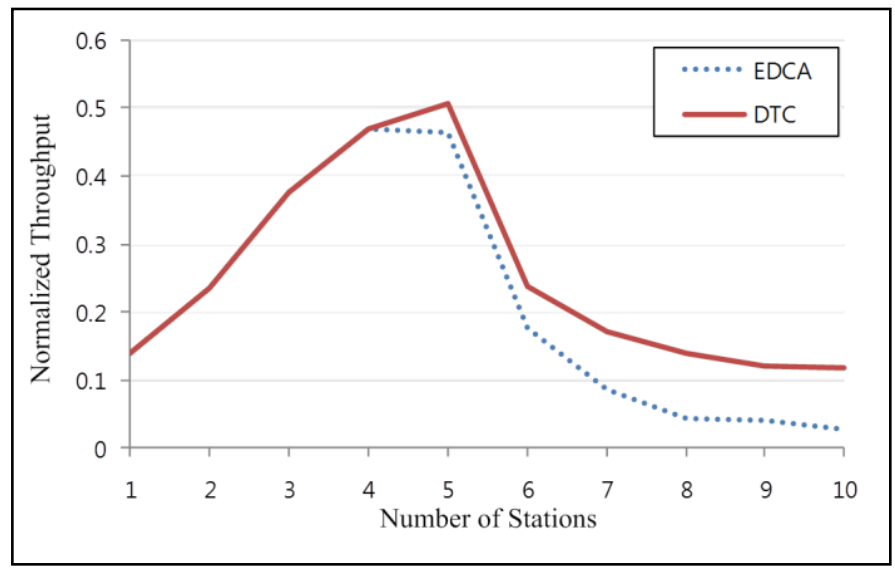

Figure 9. Normalized throughput according to the number of stations

Fig. 9 indicates normalized throughput depending on the number of stations. The throughput is calculated by considering only packets successfully transmitted within their delay bound and it is indicated in the figure through normalization. Although throughput increases similarly in both of DTC and EDCA until the number of stations reaches 4, the throughput of the DTC scheme is higher thereafter as the number of stations increases.

As shown in Fig. 8, although channel utilization is nearly same, the proposed DTC scheme further satisfies delay bound of transmitted data packets. Fig. 9 shows that our scheme is better scheme than the IEEE 802.11e EDCA. 


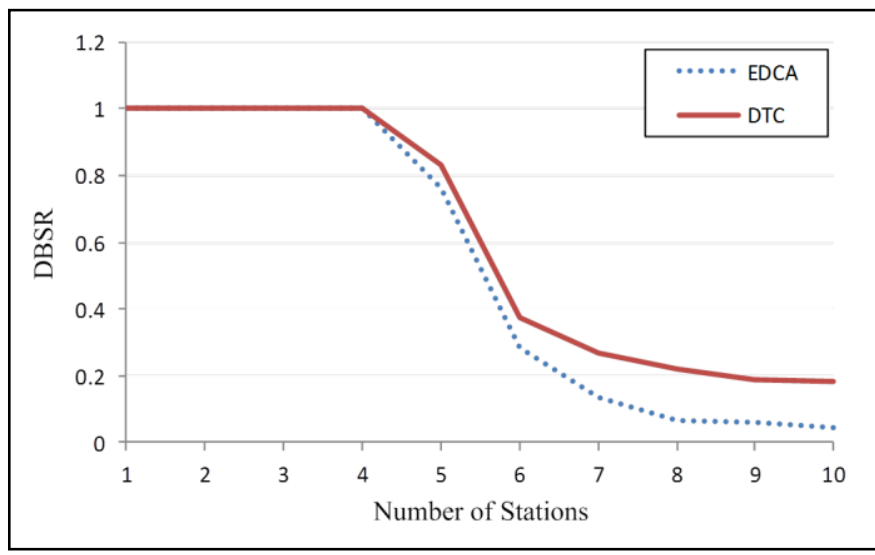

Figure 10. DBSR according to the number of stations

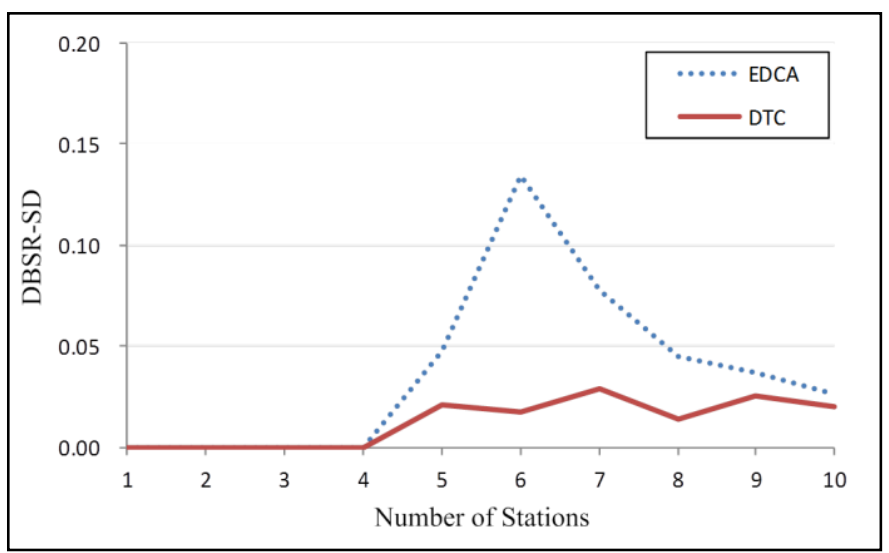

Figure 11. DBSR-SD according to the number of stations

Fig. 10 indicates the ratio of data packets which is successfully transmitted to a receiver within its delay bound. As shown in the figure, when there is small number of stations and channel utilization is low, the proposed DTC and the IEEE 802.11e EDCA have success ratio of almost $100 \%$. However, as the number of station increases, DBSR rapidly decreases due to channel congestion. The proposed DTC scheme, however, has higher success ratio than the EDCA. The high DBSR means that the quantity of packets discarded due to breaking their delay bound is low. We can see that the DTC scheme has higher performance than the EDCA when the number of stations increases.

Fig. 11 shows the results of DBSR-SD according to the number of stations. From the figure, we can see that the DBSRSD of the proposed DTC scheme is up to $10 \%$ lower than that of the EDCA. Since low standard deviation among stations implies that DBSR of each station is similar, the proposed scheme is found to be fairer in providing QoS to stations regardless of the quantity of multimedia traffic.

Fig. 12 shows the results of average queue utilization. The average queue utilization of the DTC scheme is lower than that of the IEEE 802.11e EDCA. Since the increased number of stations increases queue utilization, the DTC scheme uses bigger TXOP limit to transmit more data packets. Therefore, in the DTC scheme, multimedia traffic is transmitted faster than the IEEE 802.11e EDCA and the performance of overall network is improved.

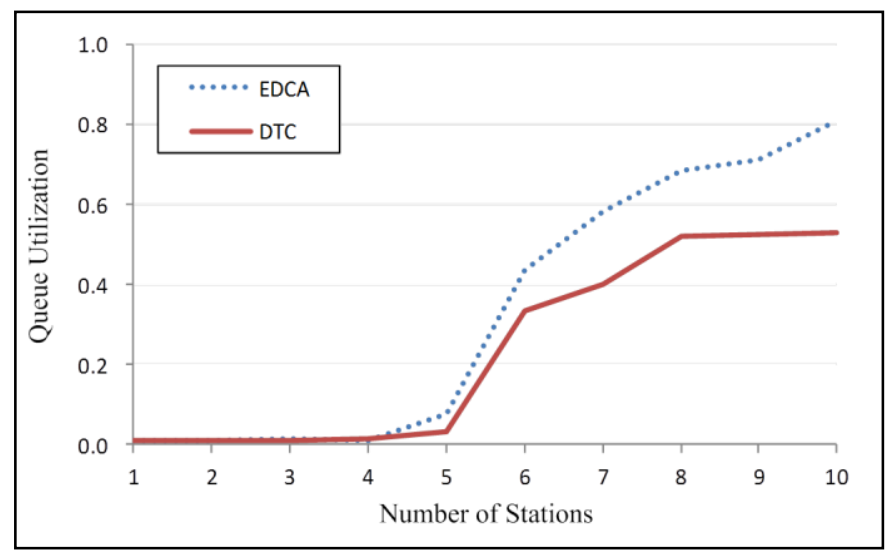

Figure 12. Queue utilization according to the number of stations

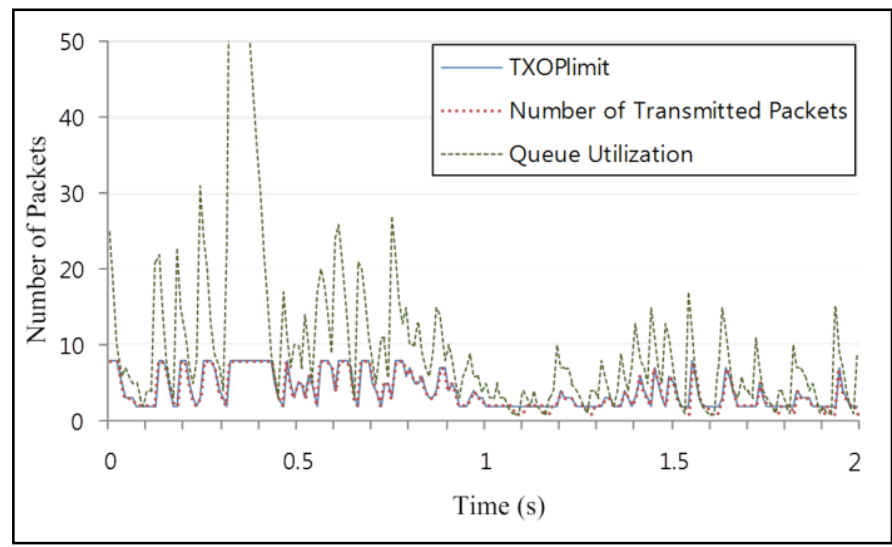

Figure 13. TXOP limit, number of transmitted packets, and queue utilization according to time in the DTC scheme

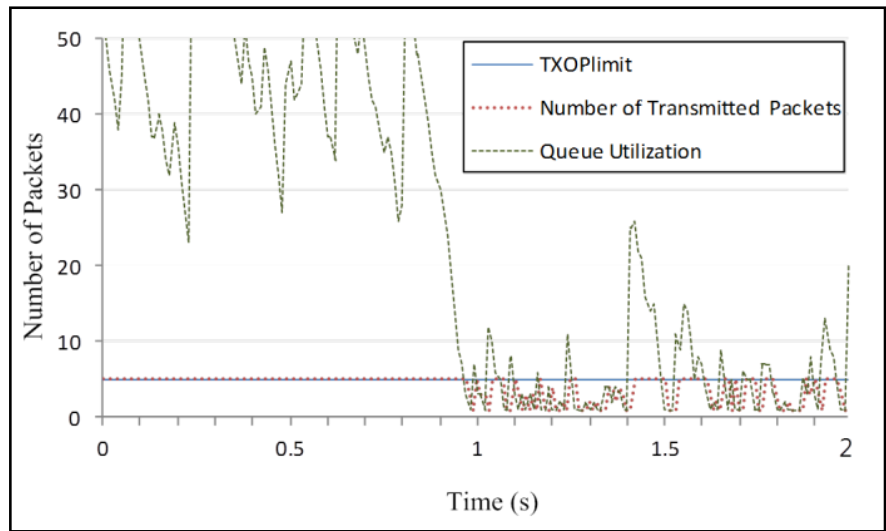

Figure 14. TXOP limit, number of transmitted packets, and queue utilization according to time in the EDCA

Figs. 13 and 14 show the results of TXOP limit value, queue utilization, and the number of transmitted packets depending on the time in one station for the DTC and the IEEE 802.11e EDCA. In the figures, the unit of queue utilization is converted to the number of packets in the queue in order to keep consistency of unit on Y axis. When there are 10 stations, one station is randomly chosen to be measured for 2 seconds. The EDCA always has fixed TXOP limit of 5 and its queue utilization is large. The DTC scheme has TXOP limit of from 2 to 8 and its queue utilization is low in average. Although TXOP $P_{Q A P \max }$ value is set to 10 in Table 1 , the DTC has TXOP 
limit value greater than or equal to 9 only when channel utilization is very low and queue utilization is very high. Thus, it cannot have such values in the situation shown in Fig. 13. The average TXOP limit value of the DTC scheme is very close to 5 and the difference in transmission possibility may be low among stations, when compared to the IEEE 802.11e EDCA.

\section{CONCLUSION}

As the IEEE 802.11e EDCA applies the same TXOP limit to stations where multimedia traffic is generated in different quantity, QoS is discriminatorily provided to each station. To alleviate this problem, we propose the DTC scheme which dynamically adjusts TXOP limit. In the DTC scheme, QAP uses channel utilization to calculate TXOP limit value, which is transmitted to each station through a beacon frame. And then, a station calculates the final TXOP limit value based on its own queue utilization information. Considering the channel utilization and queue utilization, the proposed DTC scheme adaptively allocates TXOP limit value depending on the network state to provide stations with QoS which is fairer than that provided by the IEEE 802.11e DECA. It is confirmed that the overall network performance is improved as transmission success ratio within the delay bound becomes larger.

\section{ACKNOWLEDGMENT}

This paper was supported by Research Fund, Kumoh National Institute of Technology.

\section{REFERENCES}

[1] IEEE, "Part 11: Wireless LAN medium access control (MAC) and physical layer (PHY) specifications," IEEE Standard 802.11, June 1999.

[2] Y. Xiao, "A simple and effective priority scheme for IEEE 802.11," IEEE Communications Letters, vol. 7, no. 2, pp. 70-72, 2003.

[3] H. Zhai, X. Chen, Y. Fang, "How well can the IEEE 802.11 wireless LAN support quality of service?," IEEE Transactions on Wireless Communications, vol. 4, no. 6, pp. 3084-3094, 2005.

[4] IEEE, "Part 11: Wireless LAN medium access control (MAC) and physical layer (PHY) specifications amendment: medium access control (MAC) quality of service enhancements," IEEE Standard 802.11e, 2005.

[5] S. Kim, R. Huang, and Y. Fang, "Deterministic priority channel access scheme for QoS support in IEEE 802.11e wireless LANs," IEEE Transactions on Vehicular Technology, vol. 58, no. 2, pp. 855-864, 2009.

[6] Q. Deng and A. Cai, "A TXOP-based scheduling algorithm for video transmission in IEEE 802.11e networks," Proceedings of the 6th International Conference on ITS Telecommunications, pp. 573-576, 2006.
[7] N. Cranley and M. Davis, "An experimental investigation of IEEE 802.11e TXOP facility for real-time video streaming," Proceedings of IEEE Global Telecommunications Conference, pp. 2075-2080, 2007.

[8] N.S. Shankar and M. Schaar, "Performance analysis of video transmission over IEEE 802.11a/e WLANs," IEEE Transactions on Vehicular Technology, vol. 56, no. 4, pp. 2346-2362, 2007.

[9] J. Zhu, A. Fapojuwo, "A new call admission control method for providing desired throughput and delay performance in IEEE802.11e wireless LANs," IEEE Transactions on Wireless Communications, vol. 6, no. 2, pp. 701-709, 2007.

[10] X. Chen, H. Zhai, and Y. Fang, "Supporting QoS in IEEE 802.11e wireless LANs," IEEE Transactions on Wireless Communications, vol. 5 , no. 8, pp. 2217-2227, 2006.

[11] G. Boggia, P. Camarda, L.A. Grieco, and S. Mascolo, "Feedback-based control for providing real-time services with the 802.11e MAC," IEEE/ACM Transactions on Networking, vol. 15, no. 2, pp. 323-333, 2007.

[12] S. Kim and Y.J. Cho, "Channel time allocation scheme based on feedback information in IEEE 802.11e wireless LANs," Elsevier Computer Networks, vol. 51, no. 10, pp. 2771-2787, 2007.

[13] J. Hu, G. Min, and M.E. Woodward, "A threshold-based dynamic TXOP scheme for intra-AC QoS differentiation in IEEE 802.11e networks," Proceedings of the 14th IEEE International Conference on Computational Science and Engineering, pp. 526-531, 2011.

[14] J.Y. Lee, H.Y. Hwang, J. Shin, and S. Valaee, "Distributed optimal TXOP control for throughput requirements in IEEE 802.11e wireless LAN," IEEE PIMRC, 2011.

\section{AUTHORS PROFILE}

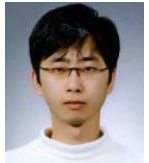

Young-Woo Nam received the B.S. and M.S. degrees in software engineering from Kumoh National Institute of Technology, Gumi, Korea, in 2010 and 2012, respectively. His research interests include wireless LANs, wireless mesh networks, quality of service enhancement.

Sunmyeng Kim received the B.S., M.S., and Ph.D. degrees in information and communication from Ajou University, Suwon, Korea, in 2000, 2002, and 2006, respectively. From May 2006 to February 2008, he was a Postdoctoral Researcher in electrical and computer engineering with the University of Florida, Gainesville. In March 2008, he then joined the Department of Computer Software Engineering, Kumoh National Institute of Technology, Gumi, Korea, as an assistant professor. His research interests include resource management, wireless LANs and PANs, wireless mesh networks, and quality of service enhancement.

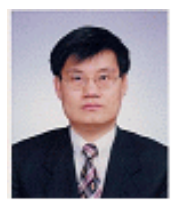

Si-Gwan Kim received the B.S. degree in Computer Science from Kyungpook Nat'l University in 1982 and M.S. and $\mathrm{Ph} . \mathrm{D}$. degrees in Computer Science from KAIST, Korea, in 1984 and 2000, respectively. He worked for Samsung Electronics until 1988 and then joined the Department of Computer Software Engineering, Kumoh National Institute of Technology, Gumi, Korea, as an associate professor. His research interests include sensor networks, mobile programming and parallel processing. 